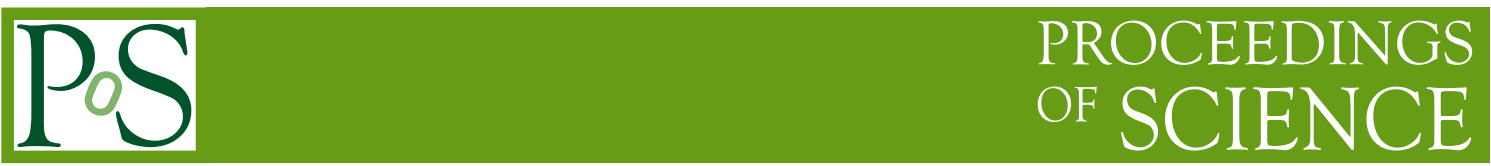

\title{
Lightning Detection at the Pierre Auger Observatory
}

\author{
Julian Rautenberg ${ }^{* a}$ for the Pierre Auger Collaboration ${ }^{b}$ \\ ${ }^{a}$ Bergische Universität Wuppertal, Wuppertal, Germany \\ ${ }^{b}$ Observatorio Pierre Auger, Av. San Martín Norte 304, 5613 Malargüe, Argentina \\ E-mail: auger_spokespersons@fnal.gov \\ Full author list: http://www.auger.org/archive/authors_2015_06.html
}

The Auger Engineering Radio Array, an extension of the Pierre Auger Observatory with antennas in the $\mathrm{MHz}$ range, requires to monitor the atmospheric conditions, which have a large influence on the radio emission of air showers. In particular, amplified signals up to an order of magnitude have been detected as an affect of thunderstorms. For a more detailed investigation and more generally, for detecting thunderstorms, a new lightning detection system has been installed at the Pierre Auger Observatory in Argentina. In addition, an electric-field mill measures the field strength on ground level at the antenna array. With these measurements, data periods affected by thunderstorms can be identified. Additionally, a lightning trigger for the water-Cherenkov detectors was developed to read out individual stations when a lightning was detected nearby. With these data, a possible correlation between the formation of lightning and cosmic rays can be investigated even at low energies of about $10^{15} \mathrm{eV}$. The structure and functionality of the lightning detection are described and first data analyses are shown.

The 34th International Cosmic Ray Conference,

30 July- 6 August, 2015

The Hague, The Netherlands

${ }^{*}$ Speaker. 


\section{Introduction}

The Pierre Auger Observatory [1], located in the Province of Mendoza, Argentina, is the world's largest cosmic-ray observatory. The objectives of the observatory are to probe the origin and characteristics of cosmic rays above $10^{17} \mathrm{eV}$ and to study the interactions of the most energetic particles observed in nature. The observatory is a hybrid system, a combination of a large surface detector (SD) and a fluorescence detector (FD). The SD is composed of 1660 water-Cherenkov stations placed on a triangular grid with nearest neighbors separated by $1500 \mathrm{~m}$. It is spread over an area of $\sim 3000 \mathrm{~km}^{2}$ and overlooked by 27 air fluorescence telescopes, as indicated in Fig. 1.

In 2006, the Pierre Auger Collaboration started an R\&D program for radio-detection of air showers [2]. With the Auger Engineering Radio Array (AERA) [3] first important results for the understanding of the radio emission process could be provided [4]. A strong enhancement of the detected amplitude in case of thunderstorms has been reported before [5, 6]. Therefore, radio detection requires the monitoring of the environmental electric field (E-field) condition to identify data periods affected by thunderstorms. This can be done using an E-field mill. In case of lightnings, the measured E-field values change within a second by several $\mathrm{kV}$. Algorithms to analyse the time-sequence of the E-field data are used to identify different environmental conditions like thunderstorm and lightning strikes [7]. The E-field mill is limited in time-precision to a onesecond sampling. Moreover, it offers no space information except for the position of the E-field mill, and its sensitivity is limited to distances of a few tens of kilometer.

\section{Lightning detector}

The StormTracker from Boltek ${ }^{1}$ is a lightning detection device which consists of a PCI card and an external antenna connected via an Ethernet cable. This device is able to detect lightning

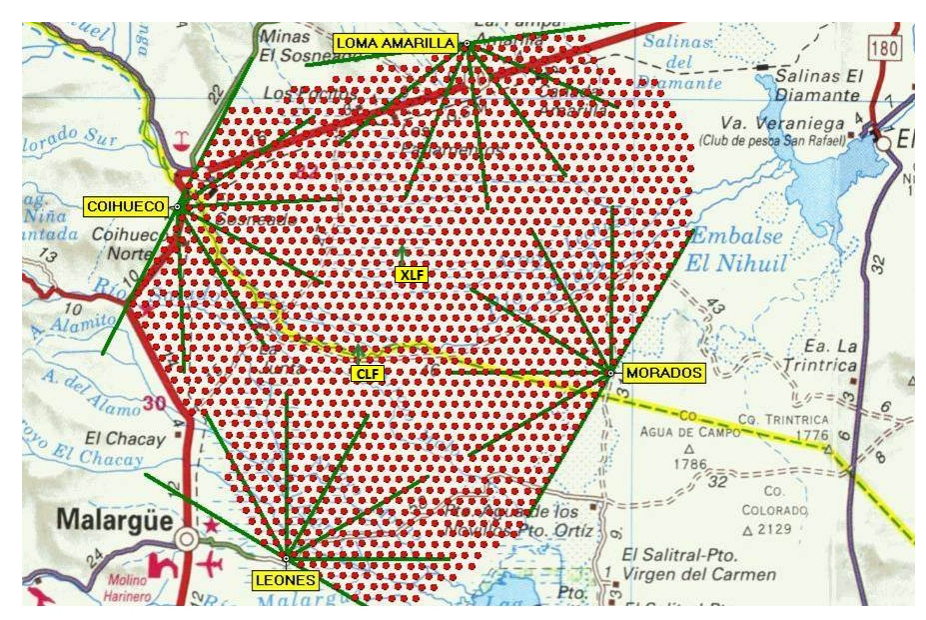

Figure 1: The Pierre Auger Observatory. Each dot corresponds to one of the 1660 surface detector stations. The four fluorescence detector enclosures are shown, each with the $30^{\circ} \times 30^{\circ}$ field of view of its six telescopes. The five lightning detector stations are located at the four fluorescence buildings and at the campus in Malargüe. From [1].

\footnotetext{
${ }^{1}$ http://www.boltek.com/product/stormpci-long-range-detection-kit
} 


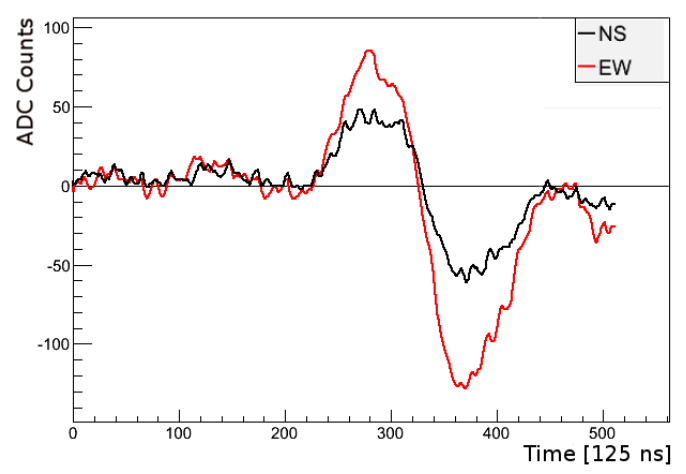

Figure 2: Example of the trace of a lightning event as detected with the Boltek StormTracker. The strong deviation from null indicates a lightning. The full length of the trace is $64 \mu \mathrm{s}$.

strikes within a radius of $1000 \mathrm{~km}$. It detects radio signals in the $\mathrm{kHz}$ region (ca. 10 to $90 \mathrm{kHz}$ ) which are initiated by lightnings.

The PCI card is mounted in a small, low-power consumption PC providing the PCI-port. The data acquisition has been programmed interfacing the Boltek Linux development kit ${ }^{2}$. This allows direct access to the PCI-card and the readout of the full sampling trace measured for the two polarization directions North-South (NS) and East-West (EW). Each input is sampled with 8 MHz. The trace contains 512 samples in a $125 \mathrm{~ns}$ binning for a full length of $64 \mu \mathrm{s}$. The card uses a threshold-trigger to generate events. An example of the two traces corresponding to the two antenna polarizations is shown in Fig. 2.

An estimation of the direction of the lightning is done by each single detector via the fraction of the signal amplitude in both polarization directions. The distance itself is estimated from the amplitude, assuming an average lightning intensity and the amplitude to scale as the square of the distance. Traces with a frequency higher than $90 \mathrm{kHz}$ are rejected as noise.

In addition to the simple position reconstruction using the polarization and amplitude of one lightning detection station (LDS) as shown in Fig. 3, the position of the lightning can be determined also from the timing information of several stations measuring the same lightning event. This coincident measurement of several stations significantly reduces the background of transient pulses mainly originating from locations close to one detector station. The necessary absolute time for the measured events is obtained by a GPS module (ublox LEA-6T ${ }^{3}$, see Fig. 4). The GPS module receives directly the trigger information from the StormTracker card via a cable. The corresponding exact absolute time can be readout from the LDS via USB.

Five LDS have been deployed at the Pierre Auger Observatory, one at the Campus in Malarguie (MG), and the other ones at each of the Fluorescence Detector buildings indicated in Fig. 1: Los Leones (LL), Los Morados (LM), Coihueco (CO) and Loma Amarilla (LA).

\footnotetext{
${ }^{2}$ previously available at http://www.boltek.com

${ }^{3}$ www.u-blox.com/de/gps-mouldes/u-blox-6-timing-module/lea-6t.html
} 

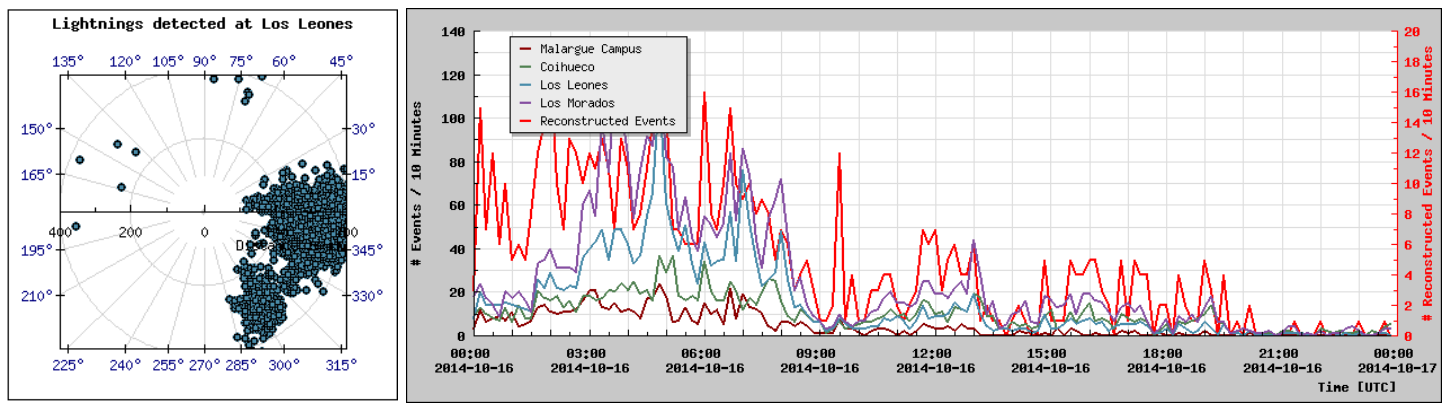

Figure 3: Left: Example of the data measured with one LDS (LL) for one day with a thunderstorm mainly east of Los Leones (16th October 2014). Right: Rates of the LDS and the reconstructed events (note the separate scale on the right).

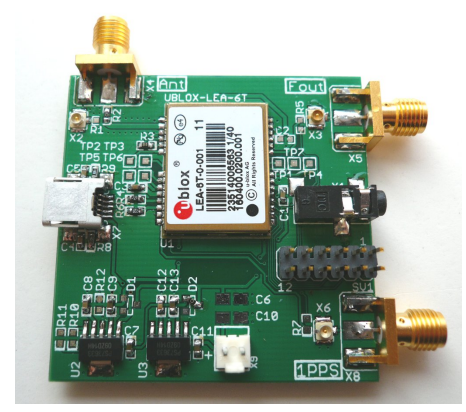

Figure 4: Picture of the GPS module integrated in the LDS and triggered by the lightning detection card.

\section{Reconstruction of lightning events}

The position of a lightning strike can be reconstructed using the absolute timing information of several stations. Considering $i$ LDS, the determination of time and place is overconstrained. It is estimated by fitting the time and place assuming the propagation with speed of light to the stations. Actually, the quantity minimized is the deviation w.r.t. one LDS, here LDS 0 is selected arbitrarily, which is compatible with minimizing to the mean time, using:

$$
t_{i}=t+\sqrt{\left(x_{i}-x\right)^{2}+\left(y_{i}-y\right)^{2}} / c .
$$

The distance from each detector to the lightning position $s_{i}$ is described as:

$$
s_{i}(x, y)=\sqrt{\left(x_{i}-x\right)^{2}+\left(y_{i}-y\right)^{2}}=s_{0}(x, y)+\left(t_{i}-t_{0}\right) \cdot c .
$$

Rearranging equation 3.2 yields $s_{0, i}$, the distance of the lightning event from detector 0 calculated using data from detector $i$ :

$$
s_{0, i}(x, y)=\sqrt{\left(x_{i}-x\right)^{2}+\left(y_{i}-y\right)^{2}}-\left(t_{i}-t_{0}\right) \cdot c
$$

Without any measurement uncertainties, $s_{0, i}$ is the same for all detectors. Since measurements do have uncertainties, the position of the lightning is estimated by minimization of

$$
P\left(x^{\prime}, y^{\prime}\right)=\min \left(\sum_{i \neq j}\left(s_{0, i}\left(x^{\prime}, y^{\prime}\right)-s_{0, j}\left(x^{\prime}, y^{\prime}\right)\right)^{2}\right) .
$$



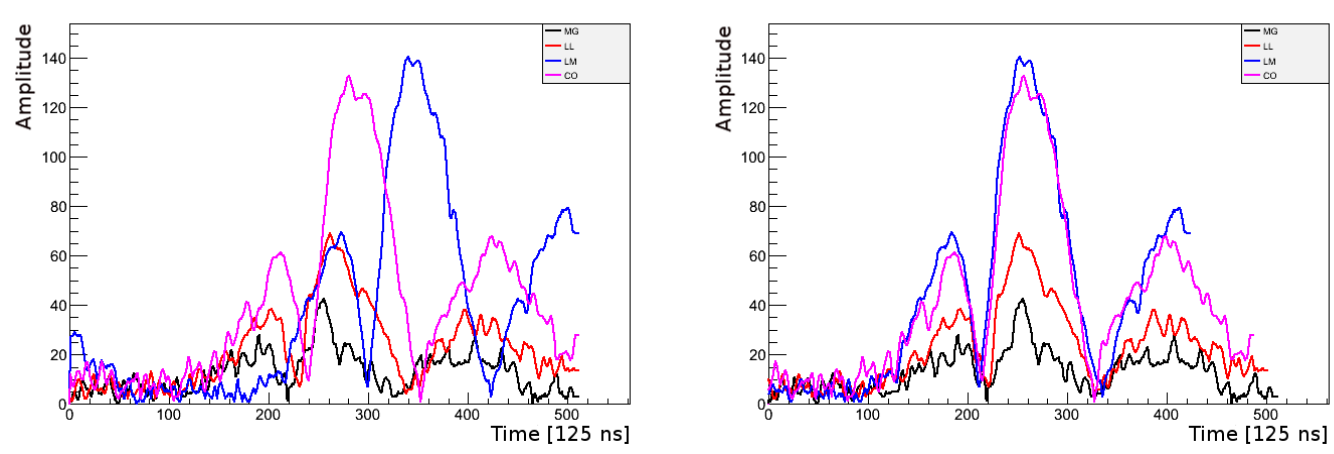

Figure 5: Example of a lightning event measured in four LDS (left). Smaller pre-peaks might exceed the threshold at a station resulting in a different reference time of the trace. The same event after shifting to maximize the cross-correlation product (right).

Important for the estimation of the lightning time and position is the exact reference time at the station. Here, a simple time of threshold is not sufficient, since lightnings often show several pulses (see example in Fig. 5), and depending on the distance, a pre-pulse can exceed the threshold while at other LDS a later pulse might be the first superating the threshold, giving different times of references for the lighting estimation. Therefore, the time of reference is estimated by maximizing the cross-correlation product, $C C$ (offset), of the full traces:

$$
\begin{gathered}
C C(\text { offset })=\max \left(\sum_{j}\left(S_{0, j} \cdot S_{i, j+\text { offset }}\right)\right) \\
\Delta t_{i}=\text { offset } \cdot 125 \mathrm{~ns} .
\end{gathered}
$$

The starting-time of each detector is corrected by adding the time offset $\Delta t_{i}$ to the start-time of the trace for the $i$ th LDS. The resulting optimal shifted traces are also shown in Fig. 5. In this way, the optimal time estimation for the different LDS independent of the amplitude of the lightning-signal is achieved.

\section{Estimation of the reconstruction accuracy}

To test the accuracy of the reconstruction method we simulated lightning events. The timing of single stations is smeared by a Gaussian distribution with a width of $30 \mathrm{~ns}$ corresponding to the absolute timing uncertainty of the GPS. The events are then reconstructed using the same algorithm. The positions of the LDS are given by the buildings of the fluorescence detectors of the Pierre Auger Observatory and the campus building. These positions are not optimally distributed, so we expect regions and directions with better and worse accuracy. In Fig. 6, the average distance between the simulated and the reconstructed position is shown for the simulated positions. Especially outside of the array, the estimation of the direction and distance are clearly different, making a polar representation more useful, as given in Fig. 7. As can be seen, the accuracy is usually about $2.5 \%$ in distance and $0.06^{\circ}$ in direction. For lightning strikes over the Pierre Auger Observatory, the position is determined typically better than $100 \mathrm{~m}$. 


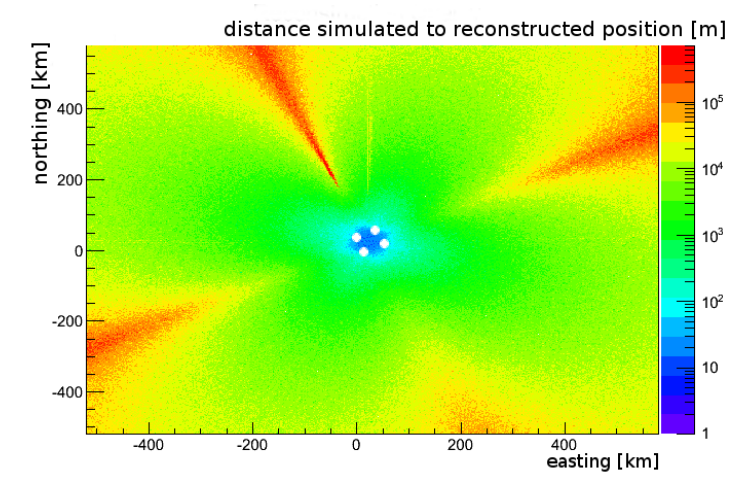

Figure 6: Average distance in meter between the simulated and the reconstructed position at the simulated position on the ground for simulated lightning events smeared according to the absolute time accuracy of the GPS modules. The four LDS used in this simulation are indicated by the white circles, which give a scale for the size of the observatory in this plot.

\section{Comparison with WWLLN data}

The WorldWide Lightning Location network (WWLLN) provides realtime locations of cloudto-ground lightning discharges [8]. Currently, more than 50 sensors [9] around the globe detect spherical activity in the VLF band. The accuracy of the network was originally determined to be about $30 \mathrm{~km}$ [10], and has been improved to $10 \mathrm{~km}$ for regions with higher sensor density. For a test of the lightning detection system at the Pierre Auger Observatory, one month of WWLLN data with lightnings occurring within $1000 \mathrm{~km}$ of the array center have been provided by the WWLLN. For the matching candidates, the difference in distance and direction are given in Fig. 8 .

\section{Prospects for cosmic ray measurement}

The additional lightning detector system at the Pierre Auger Observatory offers the possibility to study a correlation of lightnings with cosmic-ray showers. This has been an active research field in the last decade. The passage of highly localized distributions of electrons and positrons can create a region with high fractal ion conductivity in the thundercloud, which may enhance the probability to initiate the ground discharge. With the lightning system described in the previous sections, we have an accurate measurement of lightnings that can be combined with the cosmic ray measurement of the Pierre Auger Observatory at the same location.

The triggering of the surface detector of the Pierre Auger Observatory is based on a coincidence of three individual surface detector stations, since this is the minimal required measurement for a reconstruction of an air shower. Alternatively, even a single station is read out in case of a trigger based on the fluorescence detector measurement for a hybrid reconstruction. Due to the spacing of $1.5 \mathrm{~km}$ between the surface detectors, the energy threshold for air shower measurements is $3 \times 10^{18} \mathrm{eV}$ for zenith angles smaller than $60^{\circ}$. Lightnings are by far more frequent than cosmic-ray showers with energies above $10^{18.5} \mathrm{eV}$. Therefore, a correlation has to be searched for air shower below energies of $10^{18.5} \mathrm{eV}$, where the shower is too small to hit more than one surface detector. The idea is to use the lightning detection to trigger the read out of individual surface 

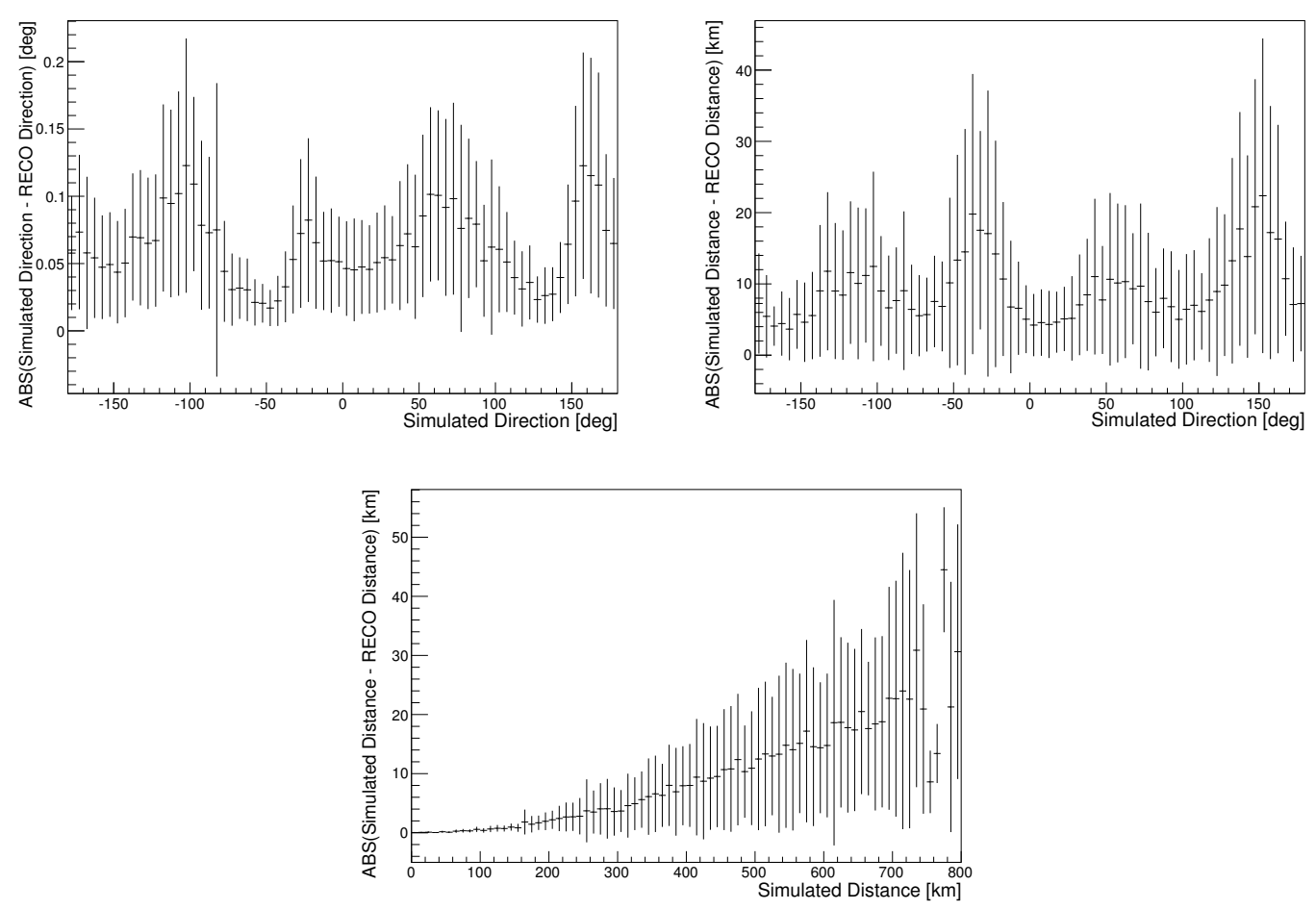

Figure 7: Reconstruction resolution for simulated lightning events smeared according to the absolute time accuracy of the GPS modules. Shown is the difference between reconstructed and simulated direction (topleft) and distance (top-right) to the array center vs the azimuth of the direction, as well as the difference in the distance vs the distance (bottom). The error-bars indicate the spread of the underlying distributions.

detector stations, in analogy of the hybrid trigger initiated by the fluorescence detector. Yet, the bandwidth to read out additional events is limited. The trigger-condition for the read out of a single station has to be restricted to a maximum window in time and space around the lightning detection that still can be transmitted by the communication system without disturbing the standard operation of the surface detector.

In addition, AERA itself is capable to measure lightning strikes with high precision due to the higher sampling rate. The operation of AERA to record lightnings is difficult in parallel to the normal cosmic ray detection, though.

\section{Summary}

The radio detection of air showers requires the measurement of the environmental electric field using a E-field mill. This allows to identify data periods affected by thunderstorms. An additional lightning detection system has been installed at the Pierre Auger Observatory to enhance the spatial and time resolution of detected lightnings. Their reconstruction is optimized using the cross-correlation of the measured signals, achieving typically $2.5 \%$ resolution in distance and $0.06^{\circ}$ in direction. This offers possible correlation studies between lightnings and cosmic rays. By providing a lightning trigger information to the data acquisition system of the surface detector, the threshold for a coincident detection of lightning and cosmic ray will be further reduced. 

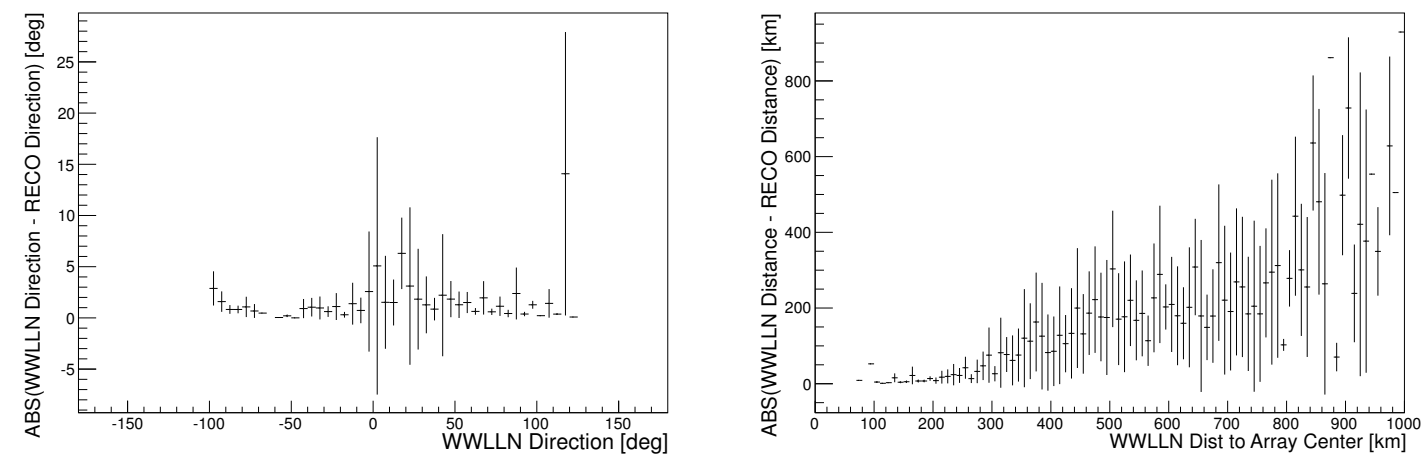

Figure 8: Comparison between the position measured with the lightning detection system installed at the Pierre Auger Observatory with the one given by the WWLLN for a sample of lightnings in October 2014. The error-bars indicate the spread of the underlying distributions.

\section{Acknowledgement}

The authors wish to thank the World Wide Lightning Location Network (http://wwlln.net), a collaboration among more than 50 universities and institutions, for providing the lightning location data used in this paper.

\section{References}

[1] The Pierre Auger Collaboration, The Pierre Auger Cosmic Ray Observatory, accepted by Nucl. Instrum. Meth. A (2015), arXiv:1502.01323.

[2] van den Berg, A. M. (for the Pierre Auger Collaboration), Radio detection of high-energy cosmic rays at the Pierre Auger Observatory, Proc. 30rd ICRC, Merida, Mexico, 2007, arXiv:0708.1709.

[3] Fliescher, S. (for the Pierre Auger Collaboration), Radio Detection of Cosmic-Ray-Induced Air Showers at the Pierre Auger Observatory, Nucl. Instrum. Meth. A 662 (2012) S124-S129.

[4] The Pierre Auger Collaboration, Probing the radio emission from air showers with polarization measurements, Phys. Rev. D 89 (2014) 052002.

[5] Mandolesi, N., Morigi, G., Palumbo, G., Radio pulses from extensive air showers during thunderstorms - the atmospheric electric field as a possible cause, J. Atmos. Terr. Phys. 36 (1974) 1431-1435.

[6] The LOPES Collaboration, Amplified radio emission from cosmic ray air showers in thunderstorms, Astron. \& Astroph. 467 (2007) 385-394.

[7] The LOPES Collaboration, Thunderstorm observations by air-shower radio antenna arrays, Advances in Space Research 48 (2011) 1295-1303.

[8] Dowden, R.L., Brundell, J.B., Rodger, C.J., VLF lightning location by time of group arrival (TOGA) at multiple sites, J. Atmos. and Sol.-Terr. Phys. 64 (2002) 817-830.

[9] World Wide Lightning Location Network, http://wwlln.net, June 2015.

[10] Rodger, C.J., Brundell, J.B., Dowden, R.L., and Thomson, N.R., Location accuracy of long distance VLF lightning location network, Ann. Geophys. 22 (2004) 747-758. 